\title{
Technology pillars for digital transformation of cities based on open software architecture for end2end data streaming
}

\author{
Cezary Mazurek \\ Poznan Supercomputing \\ and Networking Center, Poland \\ mazurek@man.poznan.pl
}

\author{
Maciej Stroinski \\ Poznan Supercomputing \\ and Networking Center, Poland \\ stroins@man.poznan.pl
}

\begin{abstract}
The goal of the paper is to expose the pillars of urban digital infrastructure and their role in the development of smart and data driven applications in the e2e model. Today, no one contests the need for a general-purpose infrastructure in the city that improves the quality of life with respect to various types of socioeconomic activity of residents. However, the digital aspect of fostering this kind of activity is linked to a very dynamic and ubiquitous element of technology, and the quality and stability of solutions based on advanced technology is a key parameter of the quality of life and development that a city should provide to its residents. This pace of technology development means that our environment (including cities) are subject to a process of continuous digital transformation. Starting from examples of such transformation and top-trends in technology development, we focus on transformation pillars that can ensure sustainable growth of innovation supply in a city. We give three examples of platforms designed for City of Poznan. We conclude that a key factor benefiting this multidimensional transformation process is the provision of an open, data-transmission and processing infrastructure with the support of which all smart city stakeholders can pursue their social, scientific, economic, or political goals in a sustainable manner.
\end{abstract}

\section{Introduction}

Currently, more than $55 \%$ of the population lives in cities. Cities will become the centers of new civilization, life and knowledge for centuries to come. This poses unprecedented challenges to the functioning of cities and their structure. It is predicted that in the perspective of 2050 it will already be 68\%. [1] In Europe, it is even estimated that by the same time almost $85 \%$ of Europeans will be living in cities. [2]

The objective of the paper is to present the pillars of digital infrastructure of cities and their role in the development of smart and data driven applications in the e2e model. Today, no one contests the need for a universal infrastructure in the city to improve the quality of life in relation to the various types of socio-economic activity of residents. However, the digital aspect of supporting this activity is linked to a very dynamic and ubiquitous element of technology, and the quality and stability of solutions based on advanced technology is a key parameter of the quality of life and development that a city should provide to its residents. [3] This dynamism of technology development makes our environment (and cities included) subject to a process of continuous digital transformation. This proces is similar to the historical building of conditions for agricultural or industrial work in the past - today, however, this supply can have a global dimension if it addresses the labor market and at the same time a local dimension when it comes to building favorable conditions for social activity, value sharing in a sustainable environment, etc. [4]

In this context, the universal 'Smart City' concept, understood as a new generation of innovative services operating within and across key areas of life, takes on a broader meaning, especially in terms of its dynamic impact on decision-making processes. Key in this respect is support in the form of platforms and technologies that support integrated analysis of data from many sources, predictive models that capture the behavior of individual urban systems as well as the interdependencies with related systems, automated system controls and interactions, and capabilities for decision-makers at all levels-from individual residents to government officials, and from small businesses to large-scale service providers-to use these integrated data sources and models to inform their own interactions and decisions [5].

In the paper, we argue that the key factor benefiting multidimensional transformation process of the city is the provision of an open, data communication and processing infrastructure with the support of which all smart city stakeholders will be able to pursue their social, scientific, economic or political goals in a sustainable manner. 
In chapter 2 , we present the smart city digital transformation models - four main trends of digital transformation strategies followed by some European cities. Than, in chapter 3 , we present top technology trends in shaping digital cities. In Chapter 4, we reference to two examples of roadmaps for digital transformation: Digital Continuum and Destination Earth. Than, Chapter 5 presents three platforms designed for City of Poznan, which illustrate how open software architecture for end2end data transmission may address digital transformation of cities.

\section{Smart City Digital Transformation Models}

Despite the considerable diversity of smart city digital transformation models, it is possible to distinguish a number of methodologies and recurring elements common to cities with similar profiles. When pointing out examples of digital transformation strategies, we focus on those that contain unique elements that distinguish them from the general ones.

The common denominator of the transformation programs is the need for strong citizen involvement. Without substantive and active involvement of citizens in the analyzed processes, any strategy, even the most intelligent and accurate one, is doomed to failure. This challenge is particularly important for models of digital transformation, whose sustainability and maintenance of smart city tools are strongly dependent on the involvement of citizens in co-creation and development. For this reason, one of the transformation tools that we have paid special attention to are the so-called living labs, whose main advantage is the possibility of methodically involving citizens in the co-creation of technological innovations already at the early stages of their design. [6][7]

Rankings of smart cities are not a reliable source of information on which cities are successfully implementing digital transformation. This results from, among others, differences in evaluation criteria of particular rankings and differences in the way indices are calculated. As a result, identifying smart cities requires adopting additional subjective evaluation criteria. We assume that the effectiveness of digital transformation is demonstrated by the effects produced in a given city, in accordance with the mechanisms clearly defined in the transformation program. Less than half of the cities listed in the rankings have strategies that contain the basic elements necessary to conduct a broadly defined digital transformation of the city. While a complete transformation programme enables a smooth launching of recommended actions, a programme being a statement of the existing state of affairs without indicating tools, areas and stages of action, is only a marketing tool.

Considering the above, for our analysis of smart city digital transformation models, we selected cities that meet the following several criteria:

- has an up-to-date digital transformation program (either as a separate document or embedded in a smart city development strategy),

- has identified areas of transformation,

- visualises a transformation model in the transformation programme,

- in the transformation program gives examples of transformation tools,

- in the transformation program gives examples of transformation projects and their results.

Four main trends of digital transformation have been identified by us, and we discuss these models further by giving examples of four cities following them. [8]

\subsection{Focused Transformation}

As an example of focused transformation, we can refer to the city of Tallin. [8] What distinguishes Tallin from other smart cities is its unique role as an experimental space, a kind of living laboratory, for the whole country. With no other large cities in Estonia, the digital transformation mechanisms there operate in a centralized model, concentrating academia, investors and pro-innovation projects around the capital. This centralization also has a feedback effect, extending the reach of implemented tools and applications to the entire country.

This situation encourages investment in egovernment tools that are low-risk to be implemented nationwide. This awareness, in turn, encourages innovators to think more broadly about the solutions they develop than just on a local scale. Observing the endogenous development of the innovation ecosystem and the growing interest of investors, also state and local governments became convinced about opening access to data, thanks to which Estonia has become a leader in implementing e-government solutions today. Furthermore, Tallin does not stop at improving the administrative processes. In the new strategy, the egovernment ecosystem has been harnessed to work on artificial intelligence in new, more developmental areas, such as airspace management for drones and the development of self-driving snow removal robots. [9] Once again, rather than addressing all technology areas, the city is clearly charting a course for its ecosystem, this time focusing on artificial intelligence. 


\subsection{Multidirectional transformation}

Among the most comprehensive and insightful, yet most popular, digital transformation programs for cities in Europe are those developed by the German Fraunhofer Institute, covered cities such as Lisbon, Chemnitz, Berlin, and Prague. [10] The program is built around three levels of analysis, the results of which capture the current state of a city and propose a coherent strategy and development plan. By combining measurable indicators with qualitative data analysis, an objective picture of city's activity is obtained. At the same time, the unique characteristics of the city that cannot be directly compared to other cities is taken into account. Although the same working methodology were used, within the program each city develops its own mechanism and unique transformation plan, including proposals for a number of smart city projects. Using Prague as an example, we can see not only the transformation tools, but also the results of their application in the form of concrete implementations.

Unlike Tallin, where the mainstream of transformation is e-government and artificial intelligence, Prague's transformation program covers all areas of city activity and many technological scopes. [11] The focal point and first stage of Prague's transformation was the establishment of a city unit dedicated to the program.

The second characteristic, dominant in the Prague program, is the focus on listening to the opinions and needs of the inhabitants. The programme assumes the launch of tools involving the inhabitants in the planning of Smart City projects, e.g. via an Internet platform.

As part of the digital transformation, Prague is implementing smart city lighting and digital city twin projects, among others. [12]

Smart lighting, includes a network of sensors installed on public light poles to improve public safety, reduction of lamp maintenance costs and data collection on noise, temperature, pollution, smart waste bins, and smart parking meters. [13] Digital Urban Twin Prague's virtualization system (Figure 1) is providing a tool for more effective control in various situations, including spread of hazardous substances, flooding and the spreading of the flood wave, urban traffic modeling and optimization, modeling the impact of urban development as well as air flows, emissions, and ventilation based on real-time display of sensor data. [14]

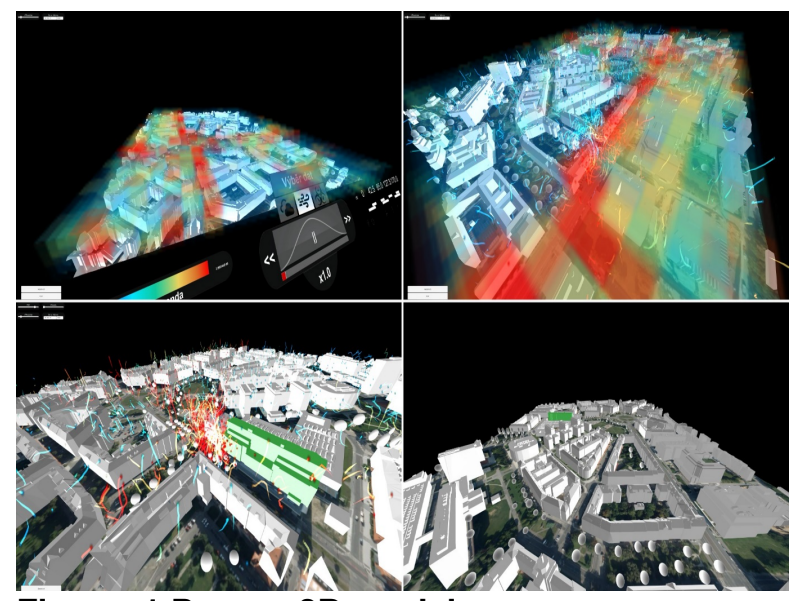

Figure 1 Prague 3D-models

\subsection{Business driven transformation}

Every smart city project is evaluated in terms of payback time and business potential. Also, the rationale for expanding the Information and Communication Technologies (ICT) infrastructure, which is the backbone of development in all areas of Tampere, is evaluated from the perspective of creating new business models and attracting private investors. [15] It should be noted that despite the strong emphasis on business aspects in Tampere's strategy, the return on investment can be obtained in values other than financial. Depending on the type of added value, other representatives of the ecosystem are responsible for implementing a given solution.

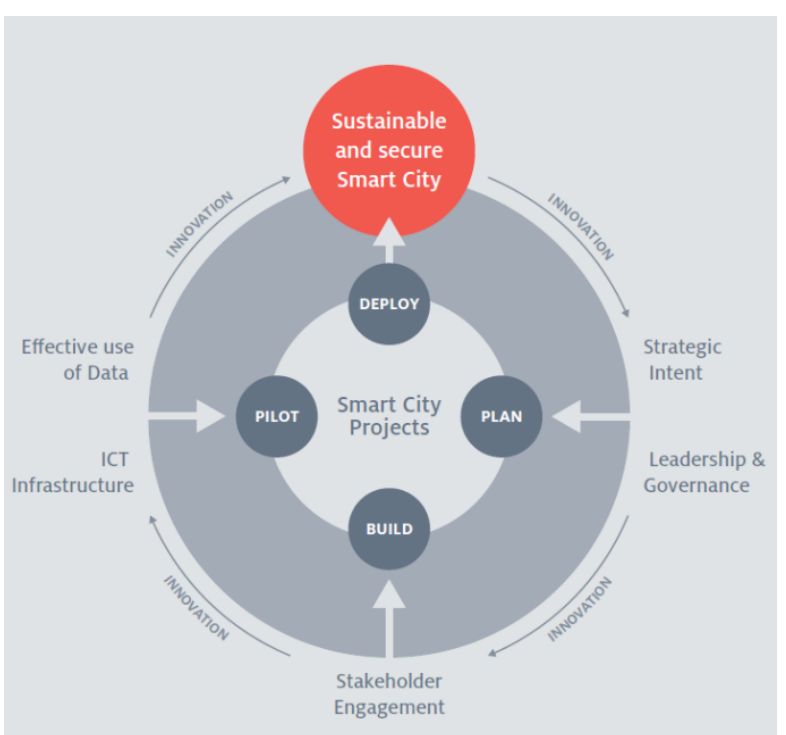

Figure 2 Smart City Program in Tampere 
The Tampere programme assumes the inclusion of the fullest possible spectrum of partners at the outset and the use of all information channels, as it is only by testing innovations that a rational business model for them can be determined and sources of funding for the production and maintenance of individual services can be defined (Fig.1). The Tampere programme assumes that the expected types of value are social, economic, community and research benefits. [16]

\subsection{SDG driven transformation}

In case of Barcelona, all areas of development of the city are implemented on two levels, a) on the level of ICT, which is the driving force and axis of integration between areas, b) on the level of the UN Sustainable Development Goals (SDG) [17], which is the reference point for monitoring progress. By placing applied technologies at the core of all other areas of the city's activity, Barcelona emphatically proclaims that the achievement of the city's strategic goals is not possible without the intensive development of AI, IoT, blockchain, robotics, photonics, 5G, big data, etc. Moreover, Barcelona's strategy adds an area of cyber security that is absent in the strategies of other cities, understood as services that protect against attacks, signal threats and protect privacy. [18]

Similar to Barcelona, Bristol is addressing the global challenges and the UN SDGs. Unlike in Barcelona, for Bristol the UN goals are not performance indicators for the city's activities, but are monitored alongside the city's own indicators. Bristol has also launched the SDG Alliance, joining the ranks of cities that are innovation labs for the SDGs and mapping global challenges to local action. Moreover, the City of Bristol animates numerous local public-private and public-public partnerships, which are called urban innovation hubs. [19] For example, the Industrial Innovation and Research Excellence Hub brings together the City of Bristol, four academic institutions and companies from the West of England Local Enterprise Association. Their combined resources allow them to create and implement innovations in numerous areas, from life sciences to energy optimization systems to intelligent transport and robotics. [20]

A comparable concept of the city as an innovation hub was presented in [21] as a framework for digital transformation focused on integration and development towards proactive smart city system. We justified there the evolutionary model of transformation by creating favorable conditions for the implementation of proof of concept projects with the active participation of residents.

\section{Top technology trends shaping digital cities}

The models outlined in the previous chapter were identified during the development of the Digital Transformation Program for the City of Poznań, for which we took as a starting point a number of observations and conclusions of a general nature. [22]

ICT are a major tool and an important focus of digital transformation. The democratization of specific areas achieved through the transformation can have a snowball effect in terms of social inclusion. For example, making a dedicated communication application available to local community leaders greatly expands the group of content creators and animators of the local innovation ecosystem. By facilitating the creation of compelling content, the city unlocks the potential of proactive citizens, who then encourage more people and communities to interact. As a result, providing a tool not only solves the current challenges of the target group, but becomes a tool to stimulate and dynamically develop the entire ecosystem. This observation is particularly true for tools and infrastructure that support data collection and analysis.

At the same time, we are seeing rapid growth in new technologies that will be relevant to urban development. These include: 5G networks, blockchain, artificial intelligence/machine learning/BigData, autonomous vehicles, low-cost airspace exploration and microsatellites, biometrics/digital IDs/digital payments, drones. [4] The explosive development of ICT (e.g. cloud computing, Internet of Things - IoT, broadband networks with "anytime, anywhere" availability, spatial processing along with $\mathrm{AR}$, VR techniques, natural language processing) has given rise to the phenomenon of the digital vortex, which, based on emerging and disruptive technologies, transforms (in the process of digital transformation) important areas of life to new dimensions.

Due to the relatively high level of readiness of the city to transform into a smart city of the future, we suggested that it should be an evolutionary one [22]. However, this is a very dangerous situation. On the one hand, not all systems operating in the city are able to provide data in the form required to implement integration processes, which in extremely difficult situations may require the exchange of these systems, and on the other hand, further development must take into account natural technological revolution described by the availability of disruptive technologies. [23]

In terms of the impact of specific technologies on the digital transformation areas out of the broad spectrum of technologies, it seems that two of them will be crucial. These are 5G (and future 6G) networks and Big Data/AI/ML processing. 


\subsection{Smart communication networks}

One of the most significant technology challenges for future smart cities is to develop a citywide network infrastructure which effectively guarantee resourceefficient and reliable communications while managing the complexity of heterogeneous devices and access technologies. [24]

In the context of the partnership which the city of Poznań has established with the PSNC, the networking infrastructure which we can look at from the perspective of its importance for the digital transformation of the city develops basing on Fiber optic network infrastructure. Fiber optic network is currently composed of two networks: the metropolitan area scientific network POZMAN and the network connecting the city's monitoring cameras. The POZMAN network in the area of the Poznań agglomeration has more than $300 \mathrm{~km}$ of own fiber optic cables connecting all scientific institutions of the city of Poznań and some schools. PSNC, as the operator of the POZMAN network, also provides a corporate network of the City Office, connecting many city units and a wireless network, on the basis of which free access to the Internet is offered. The fiber optic network for connecting cameras is being built by the Crisis Department of the City Hall. There are interrelationships between networks including exchange of fibers, pipes as well as co-investments.

\subsection{Intelligence everywhere}

Among the solutions for edge computing dedicated to network operators, the MEC (Multi-access Edge Computing) architecture proposal, which was developed by the ETSI standardization organization [25], should be singled out. The ETSI MEC solution is currently an integral part of the $5 \mathrm{G}$ network infrastructure, but it can also be used in LTE and other access networks. The MEC technique assumes the placement of computing servers within the operator's network infrastructure. MEC servers will be located at the edge of the network, i.e. near base stations or in traffic aggregation points in access networks, but will also be placed in local data centers of the telecommunications operator. The cooperation of service orchestration and computing resource management systems will enable automatic migration of services and applications (or selected components thereof) between the cloud, operator's data centers and MEC servers located at the network edge. It should also be noted that a network operator providing MEC servers in its infrastructure becomes an active participant in the provision of cloud services, in particular, in the provision of new services requiring real-time operation [26], which cannot currently be effectively provided using cloud computing resources (IoT, Edge, Cloud). An example of the concept of such integrated digital infrastructure is illustrated on Figure 3. [27]

One of the possible approaches to tackle challenges on infrastructure convergence is data-centric integration. In a digital age, data is a key resource for social and commercial activities and much of data is created or held by government. By opening up data, government can help drive the creation of innovative business and services that deliver social and commercial value. [28]

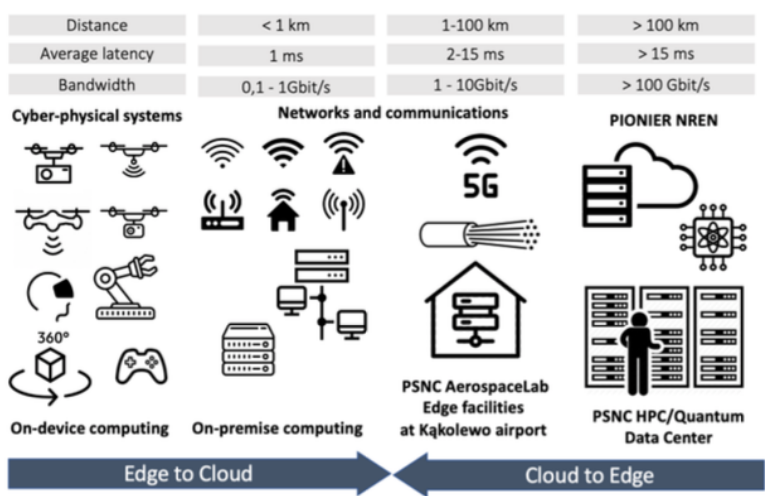

Figure 3. From edge to cloud - example of integrated digital infrastructures

\subsection{Digital Twins}

Digital Twin is a new area of technology that is an integral part of the digital transformation of the city and enables a range of research, simulation, analysis for various theses and concepts of smart city development in a virtual environment. [29] Its foundation is a digital model of a physical space that gathers information (via sensors, drones or other IoT and industrial IoT tools) and applies advanced analytics, machine learning (ML) and artificial intelligence (AI) to gain real-time insights into the performance, functioning or efficiency of a physical space. Digital Twin may also support the development towards a smarter city by helping to address critical public health, safety and environmental issues. Combining the virtual and physical worlds in this way can help better inform decision-making, reduce risk, and act as a tool for citizen engagement. A key component of a Digital Twin is the link to city governance, so that all areas related to smart city management share a common design, build, and management space, ensuring temporal and spatial continuity for all city governance processes. 


\section{Roadmaps for digital transformation}

\subsection{Digital continuum}

Technology trends presented earlier, in some specific application areas (e.g. processing data from drones) may significantly influence on the requirements for the data transmission network from the place of data generation/creation to the data center and the requirements for computing power and infrastructure of the data centers itself. Recognizing these trends, the ETP4HPC consortium introduced the idea of the Digital Continuum in which High Performance Computing plays the critical role of orchestrating and enabling workflows operated by other sister technologies such as Big Data, Artificial Intelligence, Internet of Things, etc. [30]

\subsection{Destination Earth}

Destination Earth (DestinE) is a major initiative of the European Commission. It aims to develop a very high precision digital model of the Earth (a 'digital twin') to monitor and predict environmental change and human impact to support sustainable development. To achieve this ambitious goal, the Commission is joining forces with European scientific and industrial excellence to demonstrate how digital technologies can effectively contribute to a more sustainable and digital future. [31]

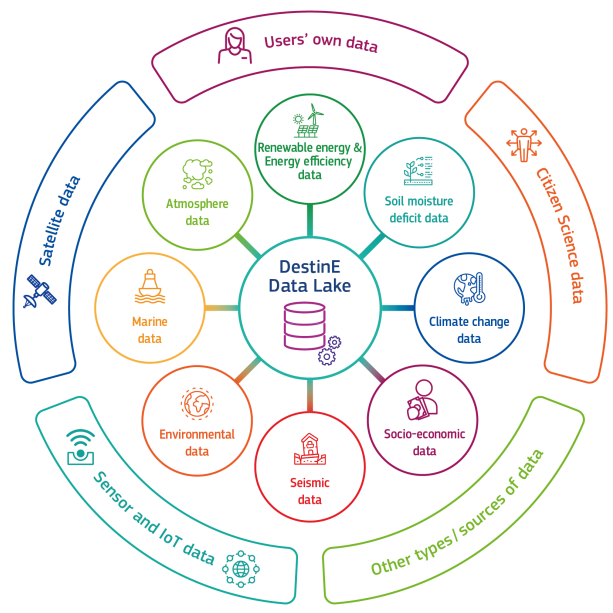

Figure 4. DestinE Data Lake

Key components of DestinE include cloud-based open core platform, data lake (Figure 4) and digital twins, which will focus on many sectors and smart cities among them.
Following both roadmaps: Digital continuum and Destination Earth we will present a concept of three platforms designed for City of Poznan, which illustrate how open software architecture for end2end data transmission is addressing digital transformation of cities.

\section{Open data transmission and processing infrastructure}

\subsection{Drone platform}

Aerial development plans and management system for this dimension of urban space is a new task for local government units within the smart city strategy. The increasingly widespread use of drones and unmanned aerial vehicles makes it imperative that in view of the regulations being created related to the so-called USPACE [32], a smart city is a city that incorporates drone traffic functions into a technically integrated vision of effective management of all components of transportation, monitoring and data acquisition. This is the task of the DRON platform. Users of the platform will include both amateurs using the airspace associated with the city (here rather the regulation will be handled by the appropriate civil aviation services) as well as city drones functioning as an infrastructure for monitoring watercourses, safety, transport or quality of green areas. In the case of this platform, special attention will be put on functions for a large scale tools for image processing and analysis, recognition of undesirable phenomena, supporting crisis management. The technology associated with drones is itself a simple and rapidly developing technology. In this context, the DRON platform provides a stable and standardized communication and information processing and monitoring service. It is a basic environment for development and applications and services for which drones will be used by residents, business and municipal services.

Naturally, there has also been an increasing need for computing power on energy-constrained computing platforms, from edge sensors to cloud and HPC systems. It was one of the most critical motivations for PSNC to set up new testing facilities called AerospaceLab in an open airfield space was to support the complex process of designing and running proofs of concept and demonstrations on a much bigger scale within natural conditions. [33]

\subsection{Open Data platform}

Thanks to the collaboration with PSNC, Poznan was the first city in Poland to introduce open data. First data 
resources were already open to citizens and business in 2011 and included not only public transportation schedules but also others, e.g. geo-data. Today, the platform includes multiple distributed data resources, including those coming from IoT-based systems such as real-time tram and bus monitoring.

The growing data has enabled us to launch efforts for advanced analysis in order to improve the functioning of various aspects of the city. Analyses concerned, for example, the use of the city bike system by citizens or the needs expressed within the projects submitted through the civic budget mechanism. The efforts are now devoted to constructing a comprehensive platform allowing to dynamically integrate, analyze and visualize city data. [22]

The integration platform prototype was developed based on OpenIoT [34] and Symbiote platforms. [35] Currently, within MARVEL project we will test a disruptive Edge-to-Fog-to-Cloud ubiquitous computing framework that enables multi-modal perception and intelligence for audio-visual scene recognition and event detection in a smart city environment. MARVEL aims to collect, analyse and data mine multi-modal audio-visual data streams of a Smart City and help decision makers to improve the quality of life and services to the citizens without violating ethical and privacy boundaries in an AI-responsible manner. [36]

\subsection{Smart Energy Platform}

A platform used for collecting, comparing and providing data on energy consumption (heating, cooling, electricity) for public institutions including grouping and comparing objects and relating the consumption to specific periods of the year or climatic conditions, expressed both in absolute and unitary values (e.g. related to the $\mathrm{m}^{2}$ of usable floor area of the object).

The platform allows for mobilization of decisionmakers to undertake actions in the field of energy efficiency, reduction of consumption by objects with high consumption, awareness of anomalies in the operation of objects in defined periods of time on a yearto-year basis or thanks to comparative analysis (e.g. noticing high energy consumption as a result of defective equipment on a year-to-year basis, noticing high energy consumption in comparison with objects of similar structure), demonstration of savings for modern or modernized objects - promotion of the use of energyefficient solutions. The effect of competition by creating a ranking and its updating in defined periods of time, defining the reward function.

The effect of the Smart Energy Platform is the improvement of energy efficiency of the objects of public institutions within the metropolis through the use of tools for monitoring heat/cold and electricity sources. For this purpose it is necessary to equip the facilities of public institutions with heat and electricity meters integrated (data tele-transmission) in the metropolitan system of supervision, comparison (benchmarking) and analysis of energy consumption. By including electricity production from RES (PV photovoltaic cells) an additional effect of RES solutions promotion would be achieved. The expected results are tools to facilitate decision making in the selection of objects for thermomodernization or installation modernization, ongoing operational analysis (identification of failures, damages, improper operation), indication of good patterns of RES use.

Additional context for the platform is related to improving the energy efficiency of public institutions in the metropolitan area through the use of innovative systems for monitoring the parameters of climatic conditions of buildings and users' activities. In this regard, the key is to use monitoring systems for climate comfort parameters (temperature, humidity, carbon dioxide $\mathrm{CO} 2$ concentration) and user activity (reed switches, occupancy sensors, counters). An additional advantage can be sensors using IoT technology, exchanging data within the Open Data Platform, with significantly lower costs compared to classical building automation systems (LON Works protocols, BacNET, $\mathrm{KNX}$, etc.). Limiting the system only to sensors significantly reduces the cost of the system (no actuators, actuators), but at the same time prevents the control at room level using BMS systems. However, in combination with applications for the involvement of environmentally conscious society, the obtained effects can be comparable to classical BACS (Building Automation and Control System) systems, and in extreme cases even more beneficial. CO2 sensors cooperating with applications of mobile devices can suggest users actions related to the quality of air in the room (operation of mechanical ventilation system, modes of operation, efficiency, replacement of filters, opening / closing windows in the framework of gravitational ventilation, etc.).

The collection of a large amount of operational data will enable the analysis of the correctness of the operation of facilities, the formulation of operational guidelines, notifications and notifications within the systems of activation of society in the field of energy savings.

\section{Conclusions}

Cities are key centers significantly influencing the pace and directions of human development. For this reason, the digital transformation of cities plays a key 
role in creating social and technological innovations that define the quality of life of future generations.

Almost three decades of successful collaboration of PSNC with the City of Poznan resulted in a number of service systems which support the municipal government and offer innovative services to the citizens of Poznan, including an official website (Multimedia City Guide).

Focusing on this partnership, we presented in this paper the pillars of digital infrastructure of cities and their role in the development of smart and data driven applications in the e2e model. We have discussed the smart city digital transformation models - four main trends of digital transformation strategies followed by some European cities. These models were identified during the development of the Digital Transformation Program for the City of Poznan. Basing on our collaboration and on the scope of this program (which introduced the concept of 29 platforms), we proposed three top technology trends shaping digital cities, namely: Smart Communication Networks, Intelligence Everywhere and Digital Twins. We find that these tree trends together have the optimal long-term innovation potential to address those global priorities. These trends may significantly influence on the requirements for the data transmission network from the place of data generation/creation to the data center and the requirements for computing power and infrastructure of the data centers itself. Therefore, as a supercomputing center and computer network operator we have focused on this three, while recognizing the potential in each of the trends mentioned in a number of different studies. Our roadmap is following strategic European initiatives: Digital Continuum as well as Destination Earth. They are key drivers for future multidimensional transformation processes and we are sure that our future work on concepts of presented platforms will be driven by these technology pillars allowing for the provision of an open, data-transmission and processing infrastructure with the support of which all smart city stakeholders can pursue their social, scientific, economic, or political goals in a sustainable manner.

\section{References}

[1] United Nations, Department of Economic and Social Affairs, Population Division (2019). World Urbanization Prospects: The 2018 Revision (ST/ESA/SER.A/420). New York: United Nations

[2] 100 climate neutral cities by 2030 - by and for the citizens. Report by the Mission Board on Climate Neutral and Smart Cities. 31 May 2020
[3] IDC. The Next Frontier: AI and Digital Twins in Smart Cities, https://blogs.idc.com/2021/07/28/the-next-frontier-aiand-digital-twins-in-smart-cities/ Accessed on 20210730

[4] Technology for smart cities: the pillars of urban planning of the future. Tomorrow City, https://tomorrow.city/a/technology-for-smart-cities-thepillars-of-urban-planning-of-the-future Accessed on 20210730

[5] Nahrstedt K., Cassandras C., \& Catlett C., "City-Scale Intelligent Systems and Platforms". arXiv preprint arXiv:1705.01990 (2017)

[6] Garcia Robles, Ana, et al. "Introducing enoll and its living lab community.", 2016, https://issuu.com/enoll/docs/enollprint? $=23453591 / 33195876$, Accessed on 20210530

[7] Citizen-Driven Innovation. A guidebook for city mayors and public administrators [Online] available at: https://openknowledge.worldbank.org/bitstream/handle/1098 6/21984/Citizen Driven Innovation Full.pdf?sequence=

Accessed on 20190606

[8] https://e-estonia.com/

[9] Report of Estonia's AI Taskforce, May 2019

[10] Morgenstadt: Creating the cities of tomorrow. https://www.iao.fraunhofer.de/en/research/urban-systemsengineering/morgenstadt-creating-the-cities-oftomorrow.html Accessed on 20210630

[11] https://www.smartprague.eu/projects/ Accessed on 20210630

[12] What "smart city" means for 2021: How digital twins, AI and other innovations drive smart transformation, Written by Richard Budden.

https://www.americancityandcounty.com/2021/06/16/whatsmart-city-means-for-2021-how-digital-twins-ai-and-otherinnovations-drive-smart-transformation/

Accessed on 20210730

[13] Why street lights could be key to building smart cities, https://www.ns-businesshub.com/business/lighting-buildingsmart-cities/ Accessed on 20210530

[14] „City Lab Prague - Executive Summary”: Alanus von Radecki, Nora Fanderl, Maike Buttler, Elena Krylova, Fraunhofer, march 2016.

[15] The Smart City Cookbook, https://nscn.eu/Tampere/SmartCityCookbook Accessed on 20210530

[16] https://smarttampere.fi

[17] 17 Sustainable Development Goals, United Nations, Department of Economic and Social Affairs. https://sdgs.un.org/ Accessed on 20190530 
[18] The Smart Cities Sector in Catalonia. Catalonia Trade \& Investment, Government of Catalonia, Barcelona, November 2019.

[19] https://www.connectingbristol.org/strategy/

[20] https://www.connectingbristol.org/wpcontent/uploads/2019/11/Connecting Bristol 300819 WEB. pdf

[21] Mazurek C., Stroiński M. “A concept of innovation hub for smart applications, enabling pro-active approach to urban policy and planning processes". Proceedings of the 53rd Hawaii International Conference on System Sciences (HICSS 2020), DOI: $10.24251 /$ hicss.2020.807

[22] Mazurek C., Stroiński M. "Common data and technological partnership - the foundation for the development of smart cities - Poznań Case Study". Proceedings of the 52nd Hawaii International Conference on System Sciences (HICSS 2019), pp 7390-7398.

[23] Radu L.D. "Disruptive Technologies in Smart Cities: A Survey on Current Trends and Challenges". MDPI Smart Cities 2020, 3, pp. 1022-1038; doi:10.3390/smartcities3030051

[24] Building a Hyperconnected City. A Global Research Initiative. ESI Thoughtlab

[25] ETSI, Multi-access Edge Computing (MEC); Framework and Reference Architecture, ETSI GS MEC 003

V2.1.1, 01.2019.

[26] P. Ren, X. Qiao, J. Chen, S. Dustdar, "Mobile Edge Computing-a Booster for the Practical Provisioning Approach of Web-Based Augmented Reality.", IEEE/ACM Symposium on Edge Computing (SEC), 2018, ISBN 978-1-5386-9445-9.
[27] Nawrocki M., Kurowski K., Gorzenski R. „Cyber space and aviation 4.0 - new testing facilities for next generation of cyber-physical, autonomous and air traffic control systems". Modern Technologies Enabling Safe and Secure UAV Operation in Urban Airspace. IOS Press 2021, pp. 70-84, DOI: $10.3233 /$ NICSP2 10008

[28] "Open Government Data" https://opengovernmentdata.org/

[29] Farsi, M., Daneshkhah, A., Hosseinian-Far, A., Jahankhani, H., "Digital Twin Technologies and Smart Cities.” Springer, Cham (2020). https://doi.org/10.1007/9783-030-18732-3 3

[30] ETP4HPC, “A blueprint for the new Strategic Research Agenda for High Performance Computing”, https:// www.etp4hpc.eu/pujades/files/Blueprint $\% 20$

document_20190904.pdf

[31]

strategy.ec.europa.eu/en/library/destination-earth

[32] https://www.sesarju.eu/U-space

[33] https://aerospacelab.psnc.pl/

[34] “OpenIoT” http://www.openiot.eu/

[35] Carozzo G., "Initial steps to build a symbIoTe enabled App", SymbIoTe Blog, March 8, 2018 https://www.symbioteh2020.eu/blog/2018/03/08/initial-steps-to-build-a-symbioteenabled-app/

[36] https://www.marvel-project.eu/ 